\title{
ORIENTAÇÃO PARA APLICAÇÃO DE SAIS DE FERRO, EM GESTANTES, SEGUNDO O USO DE CURVA OPERACIONAL DE HEMOGLOBINA ("CURVA DE UMA GRAMA") *
}

Cyro CIARI Jr.

Pedro Augusto Marcondes de ALMEIDA Alfredo ARNONI

Arnaldo Augusto F. de SIQUEIRA

R $\quad$ RSPU-B/189

CIARI JR., C. et al. - Orientação para aplicação de sais de ferro, em gestantes, segundo o uso de curva operacional de hemoglobina (Curva de uma grama). Rev. Saúde públ., S. Paulo, 7:369.75, 1973.

Resumo: Através das dosagens de hemoglobina realizadas em várias épocas da gravidez, em 553 gestantes, retiradas por amostragem casual simples de um universo de 8120 gestantes no periodo de 1947 a 1971, testou-se a aplicabilidade da curva de hemoglobina e sua importância em relação ao diagnóstico, conduta e controle do tratamento de pacientes com anemia ferropriva.

UnITERMos: Gravidez *; Assistência pré-natal *; Anemia (insuficiência de ferro) *; Curva de hemoglobina.

\section{N T RODU C A O}

Em nosso trabalho anterior 1 salientamos a importância do problema da anemia ferropriva em relação a gravidez e a oportunidade de fornecimento de suplementação de sais ferrosos às gestantes. Foi construída uma curva opera. cional com limites de tolerância, superiores e inferiores, que deveria ser testada para sabermos se ela iria ou não contribuir no diagnóstico e conduta frente a anemia e gravidez.

Este trabalho visa efetivamente evidenciar a operabilidade daquela curva, mostrando através dela se o grupo de gestantes por nós estudađo é comparável ou não ao anterior, e se portanto, esta curva pode ser utilizada para eventual conduta frente a pacientes que, embora ainda estejam com dosagens de hemoglobina, dentro de limites normais, já necessitam de suplementação férrica, e qual a época da gravidez a partir da qual estes sais ferrosos devem ser fornecidos às pacientes.

\section{MATERIAL E METODOS}

Foram observados 553 gestantes matriculadas no Serviço de Prenatal da Faculdade de Saúde Pública da Universidade de São Paulo, retiradas por amos-

\footnotetext{
* Da Disciplina de Higiene Materna do Departamento de Prática de Saúde Pública da Facul-
} dade de Saúde Pública da USP. - Av. Dr. Arnaldo, 715 - São Paulo, SP - Brasil. 
CIARI Jr., C. et al. - Orientação para aplicação de sais de ferro, em gestantes, segundo o uso de curva operacional de hemoglobina (Curva de uma grama). Rev. Saúde puibl., Paulo, 7:369-75, 1973.

tragem casual simples de um universo de 8120 gestantes no período de 1947 a 1971. Estas gestantes por suas características apresentam certa homogeneidade de condição sócio-econômica.

Estas pacientes, durante a gestação, foram submetidas a várias dosagens de hemoglobina, dosagens estas realizadas sob a mesma técnica, isto é, o da oxihemoglobina (leitura em colorímetro fotoelétrico que apresentava valores normais para as mulheres, de 13,2 a $16,7 \mathrm{~g}$ ou $83,5 \%$ a $108 \%$ ).

Destas 553 gestantes, 149 ingeriram suplemento férrico em alguma época da gestação, enquanto que 404 não receberam sais de ferro.

As várias dosagens de hemoglobina de cada uma das 553 gestantes foram inicialmente aplicadas à Figura 1 que, além de apresentar os valores médios de hemoglobina, apresenta limites de tolerância superior e inferior determinados conforme recomendam Dixon \& MASSEY ${ }^{2}$. Para cada mês de gestação foram esta. belecidos os limites que cobrem $90 \%$ da população com a probabilidade de $95 \%$. Este gráfico foi por nós elaborado ${ }^{1}$, com as 701 gestantes, e a aplicação das dosa- gens de hemoglobina das atuais 55 gestantes visa podermos estabelecer a comparabilidade destes dois grupos com a probabilidade de $95 \%$.

Além do mais anexamos a este gráfico uma outra curva operacional, sugerida pelos autores 1, curva esta construída com valores de uma grama abaixo dos valores médios da hemoglobina para cada mês de gestação e que denominamos de "curva de uma grama". (Figura 2).

Procurou-se verificar se as dosagens de hemoglobina das 553 gestantes se ajustam ou não dentro destes limites, com a finalidade de sabermos se existem pacientes que tomaram suplemento de ferro e que talvez não o necessitassem e, ao contrário, gestantes que deveriam ter recebidó o suplemento e não o receberam.

\section{RESULTADOS}

Ao aplicarmos à Figura 1 as dosagens de hemoglobina das 553 gestantes, observamos que $87,6 \%$ das pacientes apresentaram suas taxas de hemoglobina dentro dos limites de tolerância e apenas $12,4 \%$ estavam situadas fora deste limites, conforme Tabela 1 .

T A B EL A 1

Número e percentagem de gestantes que apresentaram suas taxas de hemoglobina situadas dentro ou fora dos limites de tolerância da Figura 1.

\begin{tabular}{|c|c|c|c|c|c|}
\hline Categoria & \multicolumn{2}{|c|}{$\begin{array}{l}\text { Dentro do Iimite } \\
\text { da tolerância }\end{array}$} & \multicolumn{2}{|c|}{$\begin{array}{l}\text { Fora dos limites } \\
\text { da tolerância }\end{array}$} & Tota \\
\hline $\begin{array}{l}\text { Gestantes que to- } \\
\text { maram suplemento } \\
\text { de sais de ferro. }\end{array}$ & \multicolumn{2}{|c|}{121} & \multicolumn{2}{|c|}{28} & 149 \\
\hline $\begin{array}{l}\text { Gestantes que não } \\
\text { näo tomaram suple- } \\
\text { mento de sals de } \\
\text { ferro }\end{array}$ & \multicolumn{2}{|c|}{361} & \multicolumn{2}{|c|}{43} & 404 \\
\hline \multirow{2}{*}{ TOTAL } & n. ${ }^{\circ}$ & $\%$ & n. ${ }^{\circ}$ & $\%$ & \\
\hline & 482 & 87,6 & 71 & 12,4 & 553 \\
\hline
\end{tabular}






Fig. 1 - Limites de toleráncia de Hb de $95 \%$ para $90 \%$ da populaçăo de mulheres grávidas segundo o mês de gestação e curvas operacionals sugeridas. 
CrARI Jr., C. et al. - Orientaçáo para aplicaçáo de sais de ferro, em gestantes, segundo o uso de curva operaclonal de hemoglobina (Curva de uma grama). Rev. Saride públ., Paulo, 7:369-75, 1973.

"CuRva de I grama"

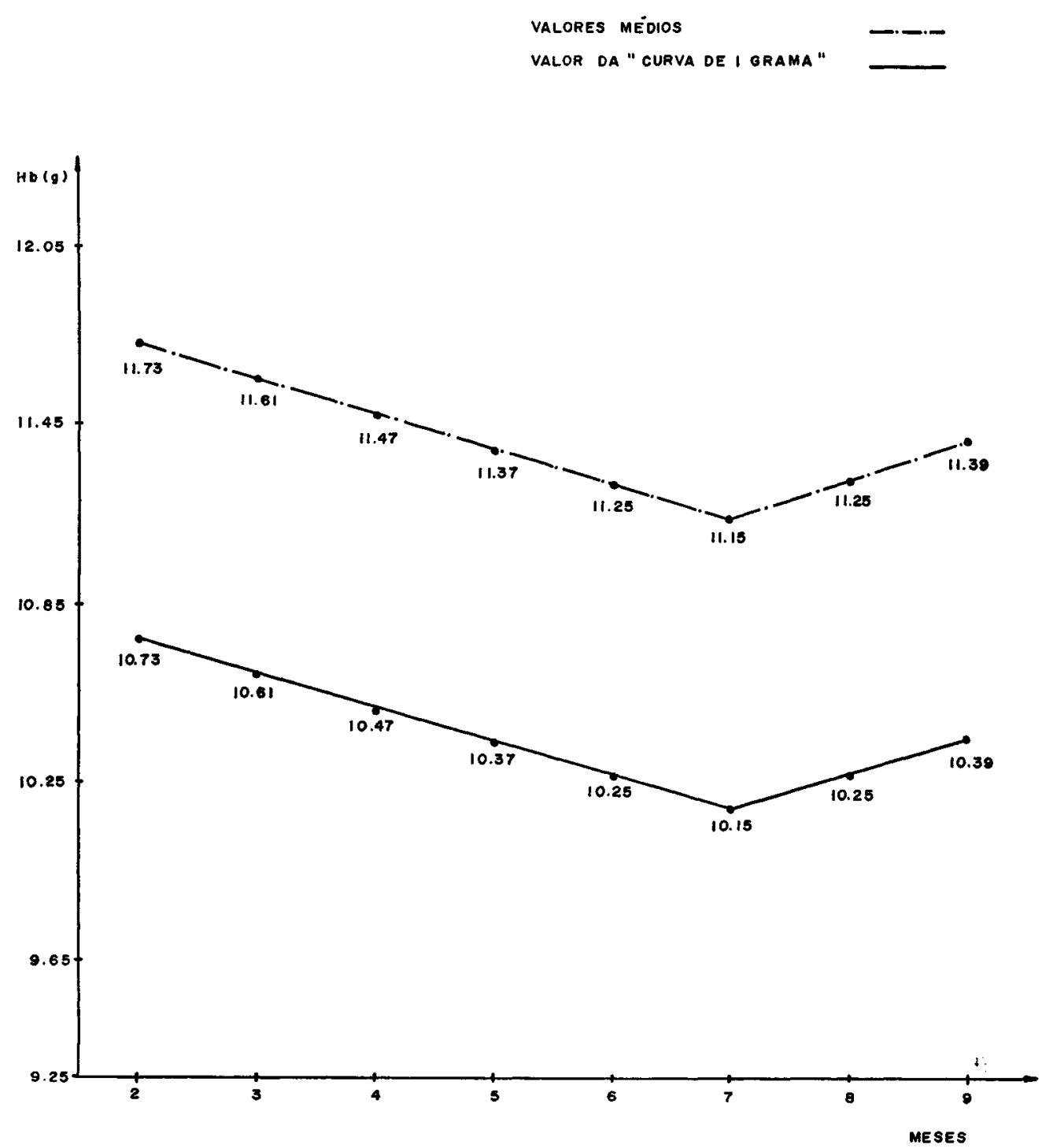

Fig. 2 - Curva de hemoglobina de 1 grama considerada como limite inferior.

Ao aplicarmos as dosagens de hemoglobina à Figura 2, porém, levando-se em conta somente a curva operacional construída com valores de uma grama ("curva de uma grama") abaixo dos va- lores médios de hemoglobina para cada mês de gravidez, observamos que das pacientes que receberam suplemento de sais de ferro (149), $73(48,5 \%)$ situavam suas dosagens, sempre acima dos limites 
CIARI Jr., C. et al. - Orientação para aplicaçáo de sais de ferro, em gestantes, segundo o uso de curva operacional de hemoglobina (Curva de uma grama). Rev. Saúde públ., S. Paulo, 7:369-75, 1973.

da "curva de uma grama", enquanto que $76(51,5 \%)$ apresentaram alguma das suas dosagens abaixo deste limite.

As gestantes que não tomaram suplemento férrico (404) se comportaram da seguinte maneira: $87(21,4 \%)$ estavam com taxas inferiores a "curva de uma grama", enquanto que $317(78,6 \%)$ apresentaram sempre dosagens acima desta curva.

TABELA 2

Número de gestantes (com ferro sem ferro) que apresentaram alguma das suas dosagens de hemoglobina acima ou abaixo da "curva de 1 grama".

\begin{tabular}{|c|c|c|c|c|c|}
\hline Categorla & $\begin{array}{l}\text { Dosagens } \\
\text { acima da } \\
\text { uma g }\end{array}$ & pre & $\begin{array}{l}\text { Dosagens } \\
\text { abaixo da } \\
\text { uma gr }\end{array}$ & das de & Total \\
\hline \multirow{2}{*}{$\begin{array}{l}\text { Cestantes que to- } \\
\text { maram suplemento } \\
\text { de sais de ferro }\end{array}$} & n. & $\%$ & $\mathbf{n} .^{\circ}$ & $\%$ & \multirow{2}{*}{149} \\
\hline & 73 & 48,5 & 76 & 51,5 & \\
\hline $\begin{array}{l}\text { Gestantes que não } \\
\text { tomaram suplemen- } \\
\text { to de sais de ferro }\end{array}$ & 317 & 78,6 & 87 & 21,4 & 404 \\
\hline Total & 890 & & 163 & & 553 \\
\hline
\end{tabular}

\section{DISCUSSAO}

Ao observarmos os resultados da aplicação da Figura 1, acrescida da "curva de uma grama", encontramos dados sugestivos e que indicam a importância desta curva de hemoglobina.

Inicialmente constatamos que das 553 gestantes, $482(87,6 \%)$ apresentaram suas dosagens de hemoglobina dentro dos limites de tolerância. Portanto, isto evidencia que com a probabilidade de $95 \%$ de segurança este grupo populacional pode ser comparado ao grupo de gestantes do nosso trabalho anterior ${ }^{1}$, sendo aplicáveis a elas todas as suas conclusões.

Através do estudo destas $\mathbf{5 5 3}$ gestantes concluimos ainda por maior importância das curvas operacionais da Figura 1. No grupo de gestantes que não tomaram suplemento de sais de ferro em nenhum momento da gestação (404), observamos que $315(78,6 \%)$ situavam suas dosagens de hemoglobina, sempre acima da "curva de uma grama" e de fato não necessitariam de suplemento férrico.
Porém, 87 gestantes, deste grupo, isto é, $21,4 \%$ apresentaram uma ou várias dosagens abaixo da "curva de uma grama", como evidencia a Tabela 3.

TABELA 3

Número de taxas de hemoglobina de uma mesma gestante que estavam abaixo da "curva de uma grama".

\begin{tabular}{c|c} 
Taxas de Hb abaixo \\
da "curva de uma \\
grama"
\end{tabular}

\begin{tabular}{cr}
\hline 5 & 2 \\
4 & 3 \\
3 & 18 \\
2 & 18 \\
1 & 46 \\
\hline & \\
\hline
\end{tabular}

Isto evidencia que das 404 gestantes que não tomaram sais de ferro 41 , isto é, praticamente $10 \%$ deveriam tê-lo feito, pois apresentaram no mínimo duas dosagens abaixo do limite minimo da "curva de uma grama". Outro fato importante 
CIARI Jr., C. et al. - Orfentação para aplicaçăo de sais de ferro, em gestantes, segundo o uso de curva operacional de hemoglobina (Curva de uma grama). Rev. Saúde públ., S. Paulo, 7:369-75, 1973.

que devemos salientar é que estas pacientes apresentavam sempre, no mínimo, duas dosagens sucessivas abaixo da "curva operacional de uma grama". Isto é de importância capital e vários autores ${ }^{1}$ são concordes em afirmar que não se deve restringir a apenas uma dosagem de hemoglobina para o diagnóstico de anemia ferropriva, mas $\operatorname{sim}$ a várias, para se ter uma evolução e podermos distinguir uma anemia efetiva de uma baixa de taxa de hemoglobina determinada apenas pela hidremia.

As restantes 46 deste grupo apresentaram apenas uma dosagem abaixo da "curva de uma grama" e, portanto, dependendo do grupo sócio-econômico, de intercorrências clínicas ou obstétricas e, principalmente, da evolução das outras dosagens em relação a curva (ascensional) não necessitariam mesmo de suplementação.

Por outro lado, no grupo de gestantes (149) que foram medicadas com ferro, observamos que $73(48,5 \%)$ estavam com dosagens sempre acima das da "curva de uma grama". Relacionando a curva de hemoglobina de cada uma destas 73 gestantes com o inicio da ingestão do ferro, concluimos que 64 pacientes iniciaram o ferro em épocas tardias da gestação $\left(8 .^{\circ}-9 .^{\circ}\right.$ mês), e que suas taxas sempre representaram uma curva estável ou que se elevava indicando a não necessidade da suplementação. Das 73 gestantes apenas 9 apresentaram curvas que, apesar de dentro dos limites, elas se achavam muito próximas do inferior podendo, neste caso, serem submeitdas ao tratamento com ferro (Tabela 4).

Ainda no grupo das gestantes que se submeteram ao tratamento com ferro, observamos que $76(51,5 \%)$, antes de iniciar a ingestão do mesmo apresentavam taxas de hemoglobina baixas e que de fato necessitavam deste tratamento.
Neste grupo constatamos o que aparece na Tabela 5.

$$
\text { TABELA } 4
$$

Comportamento da curva de hemoglobina nas 73 gestantes que apresentavam suas taxas sempre acima da "curva de uma grama".

\begin{tabular}{ll}
\hline $\begin{array}{l}\text { Pacientes com curvas decrescen- } \\
\text { tes e nos limites minimos }\end{array}$ & 9 \\
\hline $\begin{array}{l}\text { Pacientes com curvas que se } \\
\text { mantiveram estáveis }\end{array}$ & 51 \\
\hline $\begin{array}{l}\text { Pacientes com curvas francamen- } \\
\text { te ascencionais }\end{array}$ & 13 \\
\hline
\end{tabular}

TABEIA 5

Comportamento da curva de hemoglobina nas 76 gestantes, após o início do tratamento com sals de ferro.

\begin{tabular}{l}
$\begin{array}{l}\text { Pacientes com curvas descres- } \\
\text { centes após ingestão de ferro }\end{array}$ \\
\hline $\begin{array}{l}\text { Pacientes com curvas que se } \\
\text { mantiveram estáveis após a }\end{array}$ \\
ingestão de ferro \\
$\begin{array}{l}\text { Pacientes com curvas franca- } \\
\text { mente ascenstonals após in- } \\
\text { gestáo de ferro }\end{array}$ \\
\hline
\end{tabular}

Esta Tabela nos fornece a importância da aplicação da "curva de uma grama" em relação as pacientes que necessitam efetivamente da ingestão do ferro. 29 gestantes apresentaram dosagens que se mantiveram estáveis após o início do tratamento; 39 em franca ascenção e somente 8 apresentaram curvas decrescentes que, portanto, necessitavam de terem seus casos revisados, dosagens reestudadas e a observação de intercorrências, (parasitoses, hemorragias, toxemias) que apesar do ferro mantém estas dosagens em níveis baixos.

\section{CONCLUSOES}

A aplicação da "curva de uma grama" nos permite concluir: 
CIARI Jr., C. et al. - Orientaçăo para aplicaçăo de sais de ferro, em gestantes, segundo o uso de curva operacional de hemoglobina (Curva de uma grama). Rev. Saúde puibl., S. Paulo, 7:369-75, 1973.

1) quais as pacientes que independente da condição sócio-econômica, ou de intercorrência clínica ou obstétrica, devem receber sais de ferro e a oportunidade da época de início deste tratamento;

2) confirma a importância de várias dosagens de hemoglobina na gravidez, para podermos fazer o diagnóstico de anemia ferropriva e a nulidade da dosagem única, a não ser quando muito baixa;

3) a importância da aplicação de suplementação férrica em gestantes que apresentam duas dosagens sucessivas abaixo do normal;
4) aquelas que apresentam duas dosagens sucessivas, mesmo acima do limite da "curva de uma grama", mas em evidente decréscimo, devem receber suplementação de sais de ferro, pois, observamos que a espera de outra dosagem revela-nos que estará abaixo da curva;

5) revela também a importância naquelas pacientes que, submetidas ao tratamento com sais de ferro, suas taxas não se elevam evidenciando a necessi. dade da reavaliação do caso;

6) serve ainda, operacionalmente, para avaliar o resultado do tratamento.

CIARI JR., C. et al. - [The use of an hemoglobin operational curve ("One gram curve") for the iron therapy of pregnant women]. Rev. Saúde públ., S. Paulo, 7:369-75, 1973.

Summary: Through hemoglobin determinations carried out in several phases of pregnancy, in 553 pregnant women casually chosen out of a number of 8120 within the period of 1947 to 1971 , it was tested the feasibility of the hemoglobin curve previously constructed and its importance regarding diagnosis and control of treatment in patients with iron deficiency anemia.

UNITERMS: Pregnancy"; Pre-natal care ${ }^{*} ;$ Anemia, hypoferric*; Hemoglobin curve.

\section{REFERENCIAS BIBLIOGRAFICAS}

1. ALMEIDA, P. A. M. et al. - Curva de hemoglobina em um grupo de gestantes normais. Rev. Saúde pübl., S. Paulo, 7:273-82, 1973 .
2. DIXON; W. J. \& MASSEY, F. - Introduction to statistical analysis. New McGraw Hill Book Co., 1957.

Recebido para publicação em 5-9-1973 Aprovado para publicação em 9-10-1973 